\title{
MULTI-SCALE MODELING AND ANALYSIS OF CONVECTIVE BOILING: TOWARDS THE PREDICTION OF CHF IN ROD BUNDLES
}

\author{
B. NICENO*, Y. SATO, A. BADILLO, and M. ANDREANI \\ Laboratory for Thermal Hydraulics, Nuclear Energy and Safety Department \\ Paul Scherrer Institut, CH-5232 Villigen PSI, Switzerland \\ *Corresponding author. E-mail : bojan.niceno@psi.ch
}

Received October 29, 2010

In this paper we describe current activities on the project Multi-Scale Modeling and Analysis of convective boiling (MSMA), conducted jointly by the Paul Scherrer Institute (PSI) and the Swiss Nuclear Utilities (Swissnuclear). The long-term aim of the MSMA project is to formulate improved closure laws for Computational Fluid Dynamics (CFD) simulations for prediction of convective boiling and eventually of the Critical Heat Flux (CHF). As boiling is controlled by the competition of numerous phenomena at various length and time scales, a multi-scale approach is employed to tackle the problem at different scales. In the MSMA project, the scales on which we focus range from the CFD scale (macro-scale), bubble size scale (meso-scale), liquid micro-layer and triple interline scale (micro-scale), and molecular scale (nano-scale). The current focus of the project is on micro- and meso- scales modeling. The numerical framework comprises a highly efficient, parallel DNS solver, the PSI-BOIL code. The code has incorporated an Immersed Boundary Method (IBM) to tackle complex geometries. For simulation of meso-scales (bubbles), we use the Constrained Interpolation Profile method: Conservative Semi-Lagrangian $2^{\text {nd }}$ order (CIP-CSL2). The phase change is described either by applying conventional jump conditions at the interface, or by using the Phase Field (PF) approach. In this work, we present selected results for flows in complex geometry using the IBM, selected bubbly flow simulations using the CIP-CSL2 method and results for phase change using the PF approach. In the subsequent stage of the project, the importance of effects of nano-scale processes on the global boiling heat transfer will be evaluated. To validate the models, more experimental information will be needed in the future, so it is expected that the MSMA project will become the seed for a long-term, combined theoretical and experimental program.

KEYWORDS : Multi-Scale Modeling, Convective Boiling, Critical Heat Flux, Immersed Boundary Method, CIP-CSL2, Phase Field

\section{INTRODUCTION}

The worldwide effort towards a sustainable development has motivated in the last decade a revived interest in the use of nuclear power. This trend, often called the "Nuclear Renaissance" [1], has produced innovative industrial projects, which aim to cover current and future energy supply needs [2]. In parallel, energy demand has also required us to exploit in the most efficient way current installations by considering power uprate and life extension. In general, the prospective enhanced role played by nuclear energy must be based on maintaining and further improving economic competitiveness. Obviously, the highest priority will remain to guarantee the safe operation of power plants by design and monitoring procedures. A key issue for both competiveness and safety is an optimized design of the core and efficient utilization of fuel. Addressing these issues requires us to reduce excessive conservatism by developing more accurate and predictive computational methods. In particular, advanced simulation tools are necessary for nuclear reactor thermal-hydraulics, which are currently developed in a joint effort by industry and research institutions. One of the first priorities, as well as one of the most challenging and scientifically exciting tasks, is to develop predictive methods for the accurate calculation of the Critical Heat Flux (CHF), which limits the power extraction from fuel rods $[3,4]$. In fact, the current methodology used to predict CHF is still based on empirical correlations or semi-empirical models [3]. This has the consequence that large safety margins must 
be applied and new experimental data are required for new fuel assembly designs. Therefore, the development of more general predictors is driven by both safety and economic considerations. Better predictive power is expected from the use of Computational Fluid Dynamics (CFD) codes, which are increasingly being used for nuclear safety applications [4]. In these codes, mechanistic description of boiling has been recently implemented [57]. More advanced methods based on phenomenological description of boiling up to the CHF conditions have been recently proposed (e.g. $[8,9])$, which aim to be implemented in system and CFD codes. These methods rely on a variety of closure laws for bubble dynamics, and treat the heat transfer process from the rod as disjointed from the bubble hydrodynamics. A more general approach, however, requires a tight coupling between the local bubble phenomena and the resulting heat and mass transfer processes. The present authors also believe that the occurrence of the thermal crisis must naturally result from a mechanistic description of the boiling processes. As boiling, as well as a number of other issues of interest to nuclear thermal-hydraulics, is controlled by the competition of numerous phenomena at various length and time scales, a multi-scale approach has been recognized as the only effective way to tackle the problem [10-13]. The strong industrial interest in better prediction methods for $\mathrm{CHF}$ is the driving force for the current project MSMA (Multi-Scale Modeling and Analysis of boiling), conducted jointly by the Paul Scherrer Institute (PSI) and the Swiss Nuclear Utilities (Swissnuclear). This long term program will lead to prediction of high heat flux boiling and CHF. In the first phase, however, the research focuses on the low heat flux regime, where spaced columns of bubbles originate from a limited number of nucleation sites.

\subsection{General Framework}

Some progress has already been reported for the dryout type of CHF, which is of interest in Boiling Water Reactors (BWRs) [7]. The modeling of the Departure from Nucleate Boiling (DNB) type of CHF, however, still requires basic studies, because many fundamental aspects are still not well understood [14]. Theoretical studies [15] based on some experimental evidence suggest that a scale separation could make the boiling processes close to the wall at high heat fluxes and thus DNB to some extent independent of the external hydrodynamics, e.g. of the intricacies of the flow regimes. However, knowing the amount of water that can be supplied to the microlayer that wets the wall is still necessary for predicting the growth of the dry spots that eventually lead to DNB. Therefore, there is a consensus that a general predictive capability can best be achieved by properly considering all the various scales. As the Direct Numerical Simulation
(DNS) of all thermal-hydraulic processes governing CHF is beyond the capabilities of current and probably future computer power, the multi-scale approach is the only method to offer a realistic opportunity to model mechanistically the thermal crisis. The approach is based on the use of a CFD code, coupled with other computational tools that provide information on local variables that are controlled by the microscopic interaction processes close to the wall.

The definition of the scales is to some extent a matter of convenience, because the choice is linked to the specific simulation tools that are envisaged to be used to resolve the phenomena at a specific scale. Thus various boundaries have been considered [11]. In our project, we will refer to four scales:

1) Macro-scale (a few centimeters): this is the scale of the thermal-hydraulic phenomena within one or more sub-channels, and is responsible for the cross-sectional distributions of the global variables, such as void fraction, temperature, heat fluxes, fluid velocities and pressure.

2) Meso-scale (millimeters): this is the scale of the bubbles growing and detaching from the wall, where thermal and velocity fields immediately adjacent to the bubble and the wall are fully resolved.

3) Micro-scale (micrometers): this is the scale that determines the movement of the triple interline (vapor/liquid/wall) during the bubble life cycle and the evaporation of the thin liquid film (micro-layer).

4) Nano-scale (nanometers; molecular scale): this scale might be useful to analyze processes that occur at the interface between vapor and liquid, or at the triple contact line between solid vapor and liquid. The analysis at this scale would yield important parameters (such as physical properties) in the regions not amenable to measurement, but significant for the overall mass transfer problem.

Macro-, meso-, and micro-scales are shown in Figure 1. Correspondingly, the computational tools that will be developed or enhanced in the course of the present program will include:

1) A CFD code for the macro-scale. This could be a commercial CFD code or an extension for larger scale prediction of the general solver developed for DNS and Large Eddy Simulation (LES). Physical models at the CFD scale may be based on two-fluid model Reynolds-Averaged Navier-Stokes (RANS), or Lagrangian particle tracking with LES. We plan to start a $\mathrm{PhD}$ program to explore the more appropriate approach for this scale.

2) For the meso-scale, a DNS tool will be used, coupled to an Interface Tracking (IT) technique that will be used to simulate the life-cycle of a bubble and determine the heat flux from the wall, needed by the macro-scale simulations. An in-house code is currently being developed, which implements state-of-the-art numerical 
methods for efficient parallel computations. We call this tool PSI-BOIL (Parallel SImulator of BOILing phenomena); it constitutes the numerical "back-bone" of the project.

3) Treatment of the microscopic phenomena governing the evaporation rate in the micro-region, including the shape of the extended meniscus and wettability effects. For this region, a few theoretical approaches have been proposed, based on thin film theories or prescribed contact angle. Our project explores these models, but also looks at alternative ways to predict more accurately the thermal processes in the liquid micro-layer, and possibly to account for other local effects.
4) In order to treat the microscopic phenomena more accurately, we need a deeper understanding of the physical properties of water at the liquid-vapor interface. These phenomena are occurring at nanoscales and we should resort to Molecular Dynamics (MD) modeling techniques. We plan to start a Master and a Post-Doc program to explore the possibilities of MD to yield useful insights for the micro-scale.

The outline of current and future activities of the project is illustrated in Figure 2. The current phase of the project (thick frame in Figure 2) is focused on the mesoand micro-scales, and two approaches are investigated. Depending on the choice of mathematical approach, the meso- and the micro-scale could be treated either separately

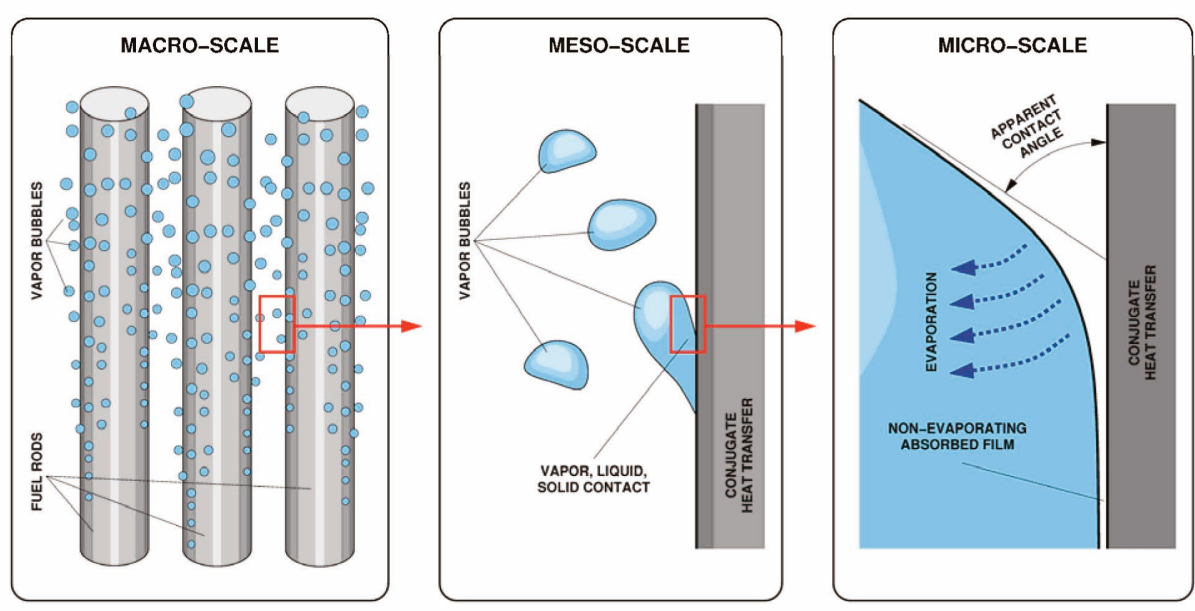

Fig. 1. Multi-Scale Concept of Boiling

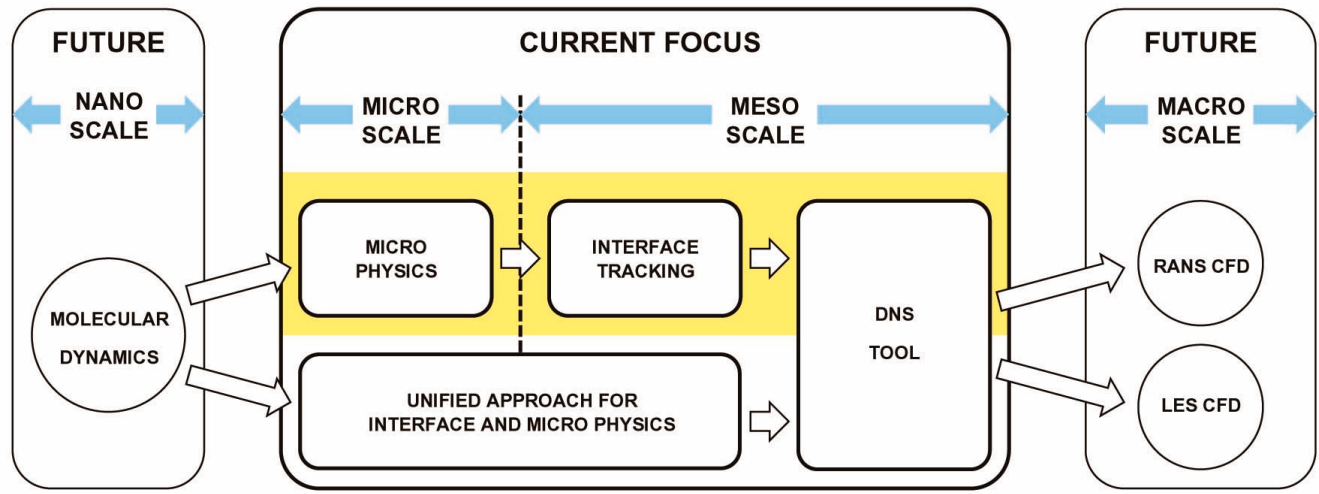

Fig. 2. Current and Future Project Activities. The Work on Micro- and Meso-Scales Has Two Branches, Separate and Unified Treatment 
(shaded area in Figure 2), or together. Keeping them separate would give freedom to chose the best model for each particular scale, but would also imply a need for coupling. On the other hand, modeling micro- and mesoscales together resolves the difficulty of coupling, but may imply higher computation costs. Therefore, at present the research program is developing along two lines:

a) development of a coupled DNS/IT tool with semiempirical treatment of the meso-scale region

b) development of a unified approach, in which the same equations are applied for both micro- and meso scales, including the bubble dynamics and the immediate vicinity to the walls.

The future work will develop towards:

c) selection of the most appropriate modeling paradigm for macro-scales, and

d) improvement of micro-scale models by MD simulations.

The comparison of results will be conducted in steps, and the final choice of method will be decided on the basis of its prospective success at predicting the hydrodynamics of a single bubble life cycle.

\subsection{Computational Tools}

The computational framework of this project is embodied in PSI-BOIL, our in-house computer program, aimed at simulation of fluid flow, heat and mass transfer phenomena at various scales. PSI-BOIL was designed to be computationally-efficient and cost-efficient for simulation of turbulent fluid flow and heat transfer phenomena. Paradigm for simulating turbulence is DNS, or LES, meaning that all, or at least most, scales of motion should be resolved on a numerical grid. The more scales of motion are resolved on available computational hardware, the more accurate results will be. Thus, efficiency of the numerical tool plays a vital role in successful simulation. The efficiency of PSI-BOIL is achieved through usage of orthogonal grids, multi-grid solution of discretized systems of equations, and parallelization for distributed memory computers. Although the usage of orthogonal grids may suggest that the program can only deal with problems in simple domains, this is not the case. An Immersed Boundary Method (IBM) has been implemented, based on cell cutting. In a practical simulation, one has to define a computational mesh in PSI-BOIL and a Computer Aided Design (CAD) file in a separate program and import the $\mathrm{CAD}$ file into PSI-BOIL. PSI-BOIL cuts the computational cells with the CAD body, creating a system of discretized equations, pertinent for geometry defined by CAD. More details on the discretization by IBM, as well as an example application, are given in Section 2.

At the beginning of the development of the IT model, we investigated several models such as the Volume of Fluid (VOF) [16] and Level Set (LS) methods [17], regarding accuracy and the implementation. The LS method was not employed because of its non-conservative properties, which violate the mass conservation law, even though the implementation and multi-dimensionalization of that method are easy compared to those in VOF. To cope with the mass conservation problem, adaptive mesh refinement techniques [18] and the hybrid particle LS method [19] are developed. However, the mass conservation problem still remains to some extent, since the distance function that is used in the LS method is basically a non conservative variable. In terms of the mass conservation, the VOF method is superior because the VOF function is the conservative variable and the mass can be exactly conserved if the conservative advection scheme is employed. Then, the VOF-PLIC (Piecewise Linear Interface Calculation) [20] and CLSVOF (coupled LS and VOF) $[21,22]$ methods are candidates; however, these schemes are difficult to implement, especially in terms of the interface reconstruction. Finally, the CIP-CSL2 (Constrained Interpolated Profile method: Conservative Semi- Lagrangian $2^{\text {nd }}$ order scheme) method [23] is selected because of its conservativeness, high accuracy, and simple implementation. In the CIP-CSL2 method, the color function is used as the phase indicator, and is advected by the flow velocities. The main feature of the CIP-CSL2 method $[24,25]$ is solving the gradient of the color function in addition to the color function itself. By using the gradient, higher accuracy can be achieved with a narrow stencil. A brief explanation and selected results of CIP-CSL2 are given in Section 3.

On the phase-change modeling side of the project, we envisioned the possibility of having a theoretical framework that would represent the physics of boiling from micro to meso-scales. This model should not only comply with conservation laws derived from a mechanistic description (i.e. jump conditions at the interface), but also should be able to provide us with additional information such as contact angle under convective conditions and even the evaporation rate at the contact line. To reach this goal, we have decided to move away from a conventional mechanistic model and follow a Phase Field (PF) approach. The roots of PF can be traced back to the $19^{\text {th }}$ century in the work of J. W. Gibbs [26], who gave a thermodynamic treatment to an interface and introduced the notion of the non-homogeneous region, modernly called the diffuse interface. Later on, in the $20^{\text {th }}$ century, Cahn and Hilliard [27] proposed a phenomenological free energy functional (very much like that of Ginzburg and Landau [28]), which coupled with a diffusion equation for a conserved order parameter, cemented the basis of modern PF models. It is thus the notion of diffuse interface and its thermodynamic description that separate PF models from mechanistic ones, in which the interface is viewed as sharp. Despite the fact that in reality any interface is diffuse (and usually extends over a few atomic or molecular radii [29]), it is clear that for diffusion controlled phase changes, the thickness of the non-homogeneous region becomes negligible compared to the extent of the boundary layer 
that controls the transformation. Therefore, from a theoretical viewpoint, a sharp interface model should be sufficient to determine the evolution of the interface due to the phase change. Nonetheless, these mechanistic models lose their validity when the thickness of the interface becomes relevant, as it is for example at the contact line, where the thickness of the extended meniscus is expected to fall within the nano-scale. Hence, a more general description of the interface, such as one based on thermodynamic grounds, should in principle be capable of representing the dynamics of the interface at the contact line. Based on these considerations, we have decided to develop a PF model, in parallel to a sharp interface formulation, to attempt a unified theoretical framework for treating simultaneously the physical phenomena occurring at micro and meso-scales.

\section{NUMERICAL METHOD}

\subsection{Orthogonal Finite Volume Method}

This section presents the essence of the numerical method we use in the PSI-BOIL program. For time discretization, the governing equations are integrated using a semi-implicit projection method [30]. The momentum equations are first solved for a tentative velocity field, discretizing the viscous terms with Crank-Nicolson scheme for stability, and the advective terms with Adams-Bashforth for simplicity. That is followed by a solution of the pressure-Poisson equation. The pressure is used to project the velocities into a divergence-free field, thus ending a time step. For spatial discretization, the governing equations are discretized on a threedimensional Cartesian grid by balancing fluxes of transported variables over the cell faces with inertial and forcing terms [31]. Momentum equations are discretized on a staggered grid for numerical robustness. Figure 3 depicts the logical coordinates and the cell face dimensions in the structured Cartesian grid. Logical indices represent cell face values if they contain the term $\delta$, or cell-centered values if they contain the term $2 \delta$.

Discretized systems of equations are solved with the diagonally preconditioned conjugate gradient method, which is further accelerated with a variant of the algebraic multigrid procedure [32], for the solution of the pressurePoisson equation.

\subsection{Immersed Boundary Method}

The immersed body is represented in our approach by its triangulated surface. For the sake of compatibility with CAD programs, we import the immersed body from a file in STereo-Lithographic (STL) format. STL files define triangulated surfaces by the coordinates of the triangle's vertices and normal surface vectors. These triangulated surfaces are used to cut the individual grid

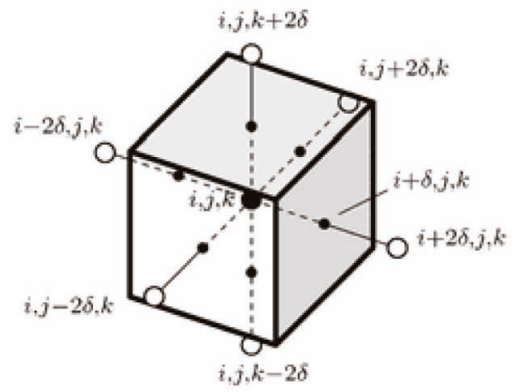

(a)

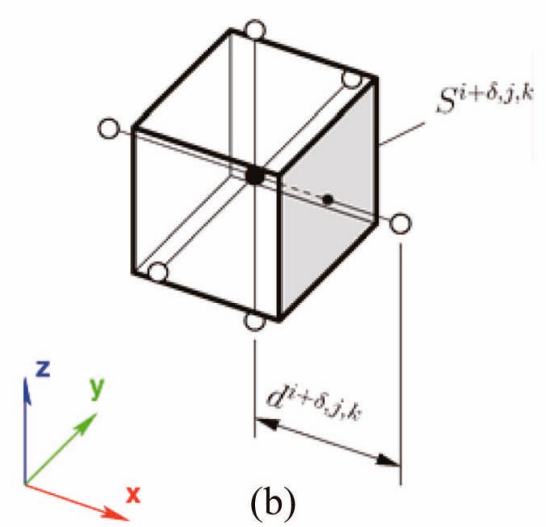

Fig 3. Logical Coordinates (a) and Cell Face Dimensions (b) in the Structured Cartesian Grid. Cell-Centered Values are Denoted with Indices Containing $2 \delta$, While the Cell Face Values Contain $\delta$. For Example, the Cell Face Between Cells $i, j, k$ and $i+2 \delta, j, k$ is Denoted by $i+\delta, j, k$

cells. Since we are using a staggered grid approach, we have four finite volume grids: one for scalar variables and one for each velocity component. To cope with immersed boundaries, we cut all three grids separately, as illustrated in Figure 4.

Cuts performed on the computational cells change their geometrical properties, and closely associated with that is the discretized system of governing equations. Geometrical quantities featured in the discretized system of equations are the cell volumes, entering the system matrix through the inertial terms, the distances between the cell centers, and the cell face areas. For evaluation of fluxes, influenced by the presence of the immersed body, two different cases have to be considered. The first occurs if both cells surrounding the face for which the diffusive flux is estimated are in the fluid. Such a situation, for the cell face $i+\delta, j, k$ is illustrated in Figure 5(a). In such a case, the distance between the cells remains unchanged, but the cell face area between them does 


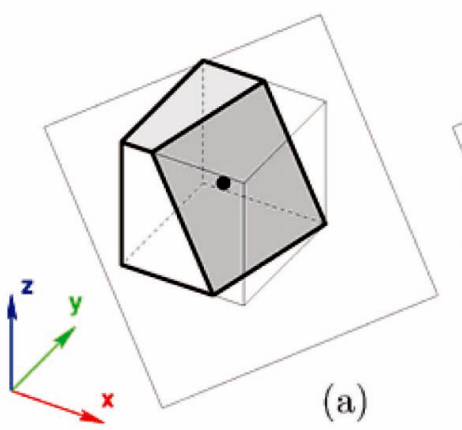

Fig. 4. Each Grid is Cut Separately: (a)

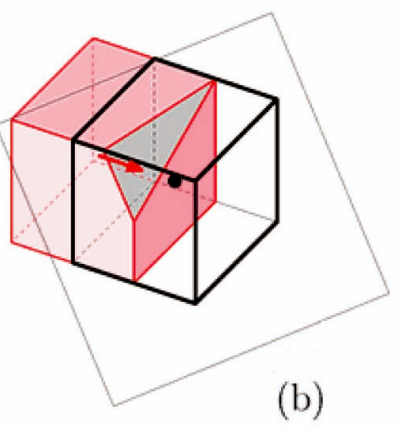

(b)

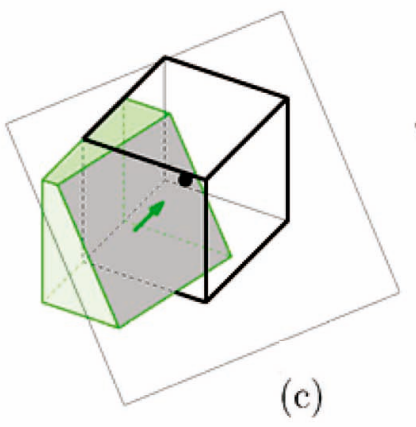

(c)

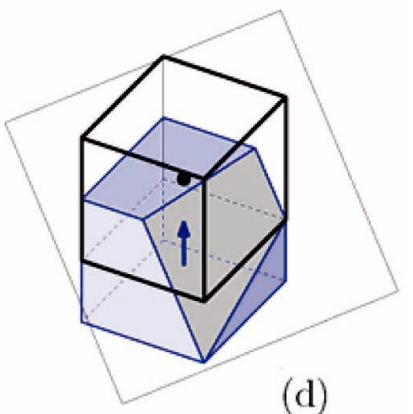

(d)

: (a) Scalar Cell, (b), (c) and (d) Momentum Cells in $x, y$ and $z$ Directions Respectively

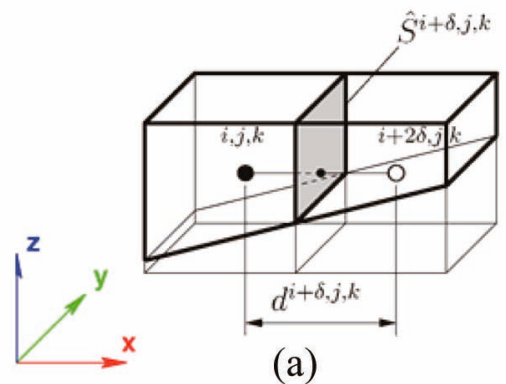

Fig. 5. Cut Cells Around the Cell Face $i+\delta, j, k$, (a) Both Cell Centers are in the Fluid, (b) and (c) Left Cell is in the Fluid, Right in the Solid change, affecting the diffusive flux through the cell face, which needs to be accounted for in the equations solved.

A different approach has to be taken if one cell, say $i+2 \delta, j, k$, is in the solid. Two such situations are illustrated in Figure 5(b) and 5(c). In such a case, the cell in the solid becomes effectively a boundary cell, and the distance between the cells decreases. That is accounted for by defining the ratio between the new and the old distances between the cells. The area is not corrected in this case. We assume that the gradient of the advected variable in the $x$ direction is constant at the face center $i+\delta, j, k$, hence the area which plays a role in the diffusive flux is the projection of the slanted surface $\left(\mathbf{S}^{i+2 \delta, j, k}\right.$ in Figure 5(b), or $\mathbf{S}^{i, j, k}+\mathbf{S}^{i+2 \delta, j, k}$ in Figure 5(c)), on the plane normal to $x$, which is simply $\mathbf{S}^{i+\delta \delta, j, k}$ on the original grid.

\subsection{Results}

In order to illustrate the validity of the numerical approach, we address a turbulent flow over a wavy surface with DNS. Compared to classical channel flow the wavy surface structure adds a degree of complexity to the flow by inducing streamline curvature, flow separation and flow reattachment, thus leading to flow situations that are also present in the flow around the fuel rod bundles. The flow was experimentally studied by Particle Image Velocimetry (PIV) in [33].

Figure 6 shows the problem domain and coordinate system and schematically illustrates the characteristic regions of the flow field in the vicinity of the wavy surface. The coordinate $x$ is directed parallel to the mean flow; $y$ is perpendicular to the top wall; and $z$ is the spanwise coordinate direction. The corresponding velocity components are denoted as $u, v$, and $w$. Characteristic regions of a flow over waves with separation, reported by [34] and [35], are the separation region (I), and the regions of maximum positive (II) and maximum negative (III) Reynolds shear stress $-\rho \overline{u^{\prime} v^{\prime}}$. The geometry of the wavy wall is determined by the ratio of the amplitude, $2 a$, to the wavelength, $\Lambda$ :

$$
\alpha=\frac{2 a}{\Lambda}
$$


and the flow is characterized by the Reynolds number, defined as:

$$
\operatorname{Re}_{h}=\frac{U_{B} \cdot h}{v},
$$

where $v$ denotes the kinematic viscosity, $h$ is the half-height of the channel, and $U_{B}$ is the bulk velocity.

To validate the DNS results, computed profiles are plotted against the PIV measurements at different streamwise locations $x / H$. Here $x / H=0.00$ and $x / H=1.00$ denote the wave crest, $x / H=0.50$ the wave trough, and $x / H=0.25$ and $x / H=0.75$ the inflection point of the wall profile. Figure 7(a) depicts the comparison of the mean streamwise velocity profiles for $R e_{h}=11200$. A good agreement between experiments and simulations is obtained for the mean streamwise velocity component at all locations. Figure 7(b) depicts the comparison of the streamwise stress profiles for the same $R e_{h}$. Also, this comparison reveals a good agreement between the experiment and the simulation for the turbulent statistics. Therefore we conclude that the DNS approach with the IBM method performs well in the prediction of turbulent separated flows over wavy walls, such as the ones encountered in flow around fuel rod-bundles.

\section{INTERFACE TRACKING WITH CIP-CSL2 METHOD}

This section presents the brief introduction of the CIP-CSL2 method and several sample calculations of two phase flow. The results presented below are obtained with the implementation of the CIP-CSL2 method in PSIBOIL code.

\subsection{The Method}

The Cubic Interpolate Profile (CIP) method, developed by Yabe $[24,25]$, is a scheme for solving hyperbolic equations, especially advection equations, for the color function or the density function. In this method, the gradient of the color function is calculated in addition to the value of the color function itself. The main strength of the CIP method is the low numerical diffusivity and stability of the solution procedure, obtained on a compact stencil. The original CIP method is non-conservative [24, 25], which is its major drawback, so a conservative CIP method was proposed later. The conservative scheme is abbreviated CIP-CSL (Conservative Semi-Lagrangian) and the different abbreviations are used in accordance with the accuracy in space; CIP-CSL2 is the second order scheme [23], CIPCSL3 is the third order [36], and CIP-CSL4 is the fourth order scheme $[37,38]$. These schemes are applied to various

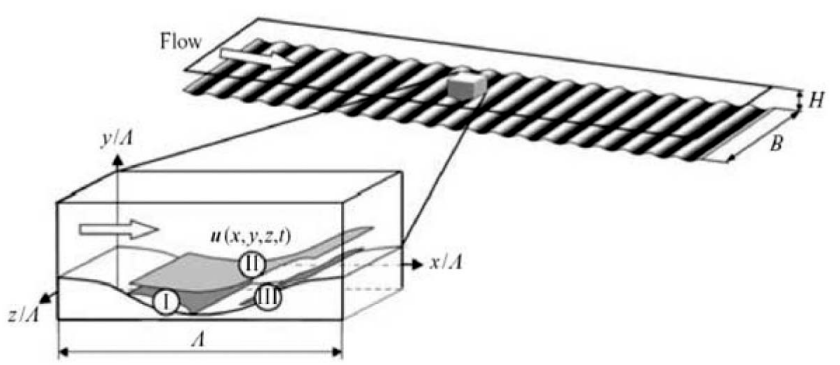

Fig 6. Problem Domain, Coordinate System and Characteristic Flow Regions

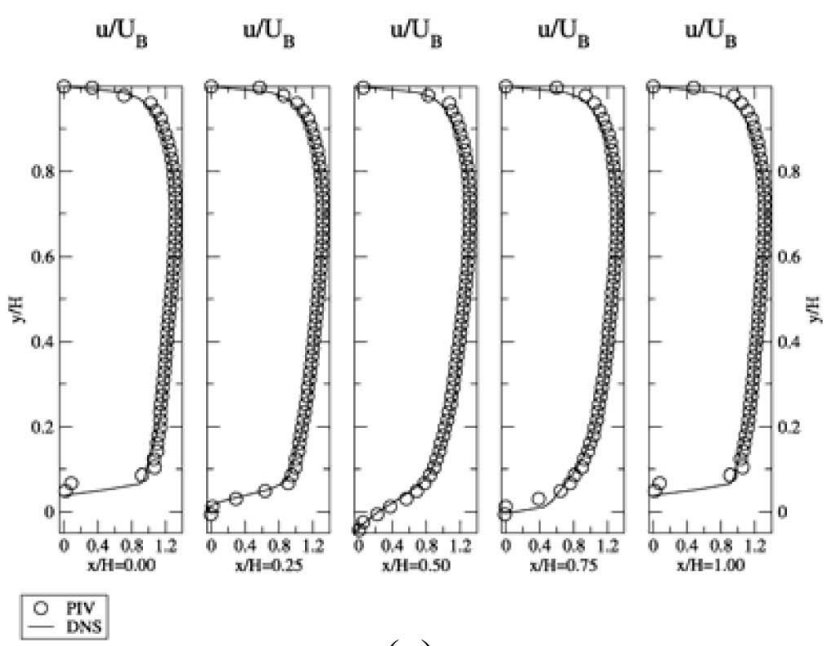

(a)
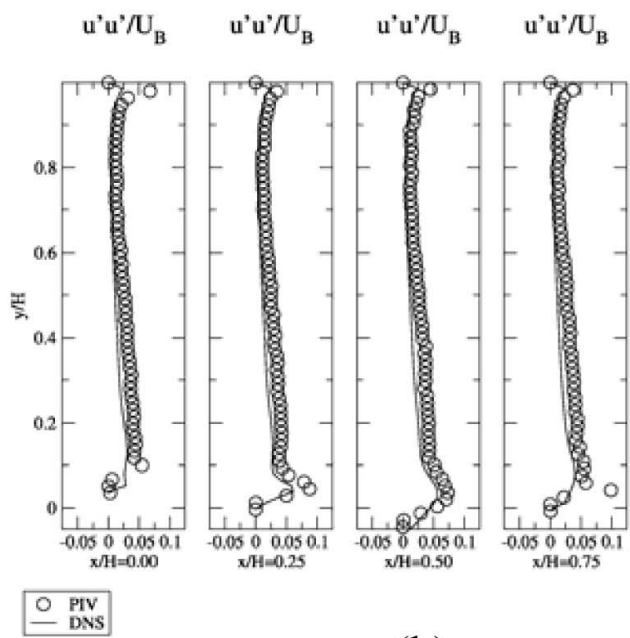

$u^{\prime} u^{\prime} / \mathrm{U}_{\mathrm{B}}$

(b)

Fig 7. Comparison of (a) Mean Streamwise Velocity Profiles and (b) Streamwise Velocity Fluctuations Between PIV (Symbols) and DNS (Lines) 
complex fluid flow problems, such as the milk crown [39], the shallow water equations [40], and the structure-fluid interaction problem [41]. In these applications, the scheme showed high accuracy with exact mass conservation. In addition to its superior performances over original CIP, CIP-CSL2 implementation is straightforward, using the directional split method, as described in [23]. Furthermore, the scheme is appropriate for parallel computation, since the stencil is compact.

\subsubsection{Mathematical Formulation}

CIP-CSL2 is a method for computing an advection equation. In the conservative form, the advection equation of a scalar variable $f$ is written as:

$$
\frac{\partial f}{\partial t}+\nabla \cdot(f \vec{u})=0,
$$

where $\vec{u}$ is the velocity vector of the advection. Onedimensional equation is explained here, for the sake of clarity:

$$
\frac{\partial f}{\partial t}+\frac{\partial(f u)}{\partial x}=0
$$

In the CIP-CSL2 method, the integral value $D$ of the variable $f$ is introduced:

$$
D=\int_{x} f d x
$$

It is clear that the derivative of $D$ is $f$ :

$$
\frac{\partial D}{\partial x}=f
$$

Here, we introduce the following equation:

$$
\frac{\partial D}{\partial t}+u \frac{\partial D}{\partial x}=0
$$

Note that the derivative of Eq. (7) with regard to $x$ is:

$$
\begin{aligned}
& \frac{\partial}{\partial x}\left(\frac{\partial D}{\partial t}+u \frac{\partial D}{\partial x}\right)=\frac{\partial}{\partial x}\left(\frac{\partial D}{\partial t}\right) \\
& +\frac{\partial}{\partial x}\left(u \frac{\partial D}{\partial x}\right)=\frac{\partial f}{\partial t}+\frac{\partial(f u)}{\partial x}=0,
\end{aligned}
$$

which is coincident with Eq. (4). Equation (5) governs the transport of a derivative of variable $f$ and, since it is in non-conservative form, can be solved with the original CIP method. Detailed description of the CIP method is given in [24].

\subsubsection{Multi Dimensional Oscillation Preventing Method}

In order to prevent numerical oscillation, the rational CIP-CSL2 method [23, 42], in which a rational function is used instead of the cubic function to prevent numerical oscillation, is employed. For three-dimensional calculation with the CIP-CSL2 method, the directional splitting method [23] is used. In the directional splitting method, the advection equation is solved in $x, y$, and $z$ directions in turns.

\subsubsection{CIP-CSL2 for Two-Phase Flow}

The mass conservation equation for the two-phase flow is defined as:

$$
\frac{\partial \rho}{\partial t}+\nabla \cdot(\rho \vec{u})=0
$$

In the following, we call the phases air and water for simplicity. The color function $\varphi$ is introduced as a volume fraction of the water inside a control volume and is defined as:

$$
\varphi=\left\{\begin{array}{cc}
0 & \text { full of air } \\
0<\varphi<1 & \text { including air and water } \\
1 & \text { full of water }
\end{array}\right.
$$

The averaged density of the control volume is defined as:

$$
\rho=\varphi \rho_{W}+(1-\varphi) \rho_{A}
$$


where $\rho_{w}$ and $\rho_{A}$ are the density of water and air, respectively. Substituting Eq. (11) into Eq. (9), the following equation can be obtained:

$$
\left(\rho_{W}-\rho_{A}\right) \frac{\partial \varphi}{\partial t}+\left(\rho_{W}-\rho_{A}\right) \nabla \cdot(\varphi \vec{u})+\rho_{A} \nabla \cdot \vec{u}=0
$$

Using the assumption of incompressible flow, the governing equation for the color function can be defined as:

$$
\frac{\partial \varphi}{\partial t}+\nabla \cdot(\varphi \vec{u})=0
$$

The CIP-CSL2 method is used to solve Eq. (13).

As a surface tension model, the Continuum Surface Force (CSF) model [43] is employed, for simplicity.

\subsection{Results}

Two cases of the two-phase incompressible flow computation are carried out as sample calculations of CIP-CSL2: the coalescence of two bubbles, and a multiple rising bubbles simulation. Note that the summation of the color function in the computational domain can be kept exactly constant during the computation for all calculations, since CIP-CSL2 is a conservative method.

$$
\sum \varphi V_{\text {cell }}=\text { const. }
$$

where $V_{\text {cell }}$ is the volume of the computational cell.

\subsubsection{Coalescence of Two Bubbles}

The coalescence of two bubbles is computed as a validation of the topology change of the free-surface. The computed results are compared with the experimental data of Brereton [44]. The computational domain is given by $-0.03 \leq x \leq 0.03,-0.03 \leq y \leq 0.03$, and $0 \leq z \leq 0.06$ and the $z-$ axis points upward. The equal spacing grid is used and the number of cells is $192 \times 192 \times 384$ in $x, y$, and $z$-direction. Two spherical bubbles of $0.01(\mathrm{~m})$ diameter are released from the positions $(0,0,0.025)$ and $(0.008,0,0.0125)$. The Eötvös number, Eo, and the Morton number, $M o$, are set as the same values as those in the experiment: $E o=16$, $M o=0.0002$.

The comparison of the bubble shapes between the computation and the experiment is shown in Figure 8. Since the initial bubble shape of the computation is spherical and is different from bubble shape of the experiment, it is not reasonable to compare the details between the computation and the experiment. Broadly speaking, the computation can predict the motion of the trailing bubble well; the trailing bubble once goes to the region below the leading bubble, and then it rises upward and catches up with the leading bubble.

\subsubsection{Multiple Rising Bubbles Simulation}

Fifty air bubbles are initially placed in water in a random pattern, as shown in Figure 9(a). The diameter of the spherical bubble is $5(\mathrm{~mm})$ and the computational domain has the dimensions of $40 \times 40 \times 80(\mathrm{~mm})$ in length, breadth, and height, respectively. The equal spacing grid is used and the number of cells is $128 \times 128 \times 256$. The side boundaries are the wall with no-slip condition; the top and the bottom boundaries are the periodic boundary condition. The density of air is $1.25\left(\mathrm{~kg} / \mathrm{m}^{3}\right)$, and of water $1000\left(\mathrm{~kg} / \mathrm{m}^{3}\right)$. The dynamic viscosity of the air is $1.0 \times 10^{-5}(\mathrm{~Pa} \mathrm{~s})$, and of water, $1.0 \times 10^{-3}$ (Pa s).

Initially, the bubbles start to rise only due to the buoyancy force. That is followed by bubble deformation and coalescence. Larger bubbles are formed in accordance with the time advancing, and the number of bubbles
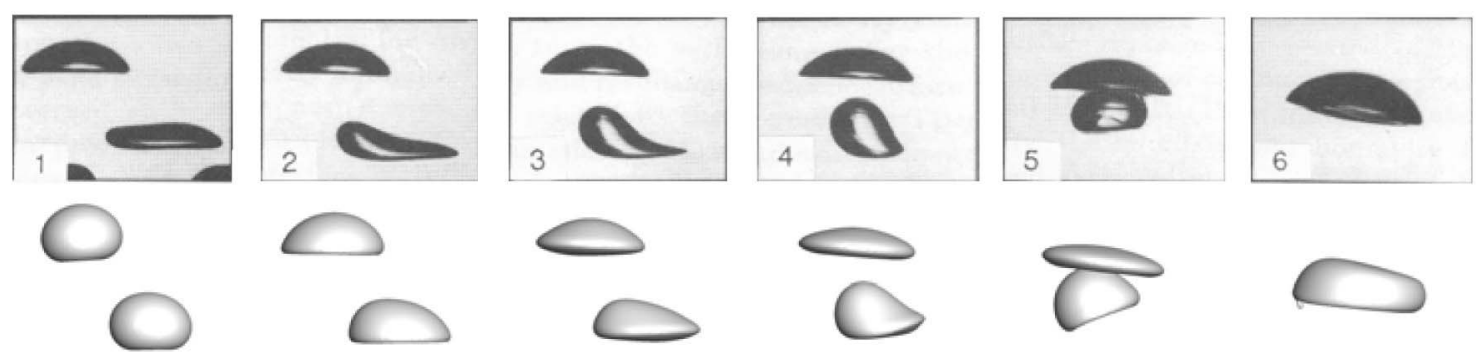

Fig. 8. Sequential Snapshot of Oblique Coalescence of Experimental Data (Top) and Computed Results (Bottom). Time Difference Between the Frames is 0.03 (s). $E o=16, M o=0.0002$ 


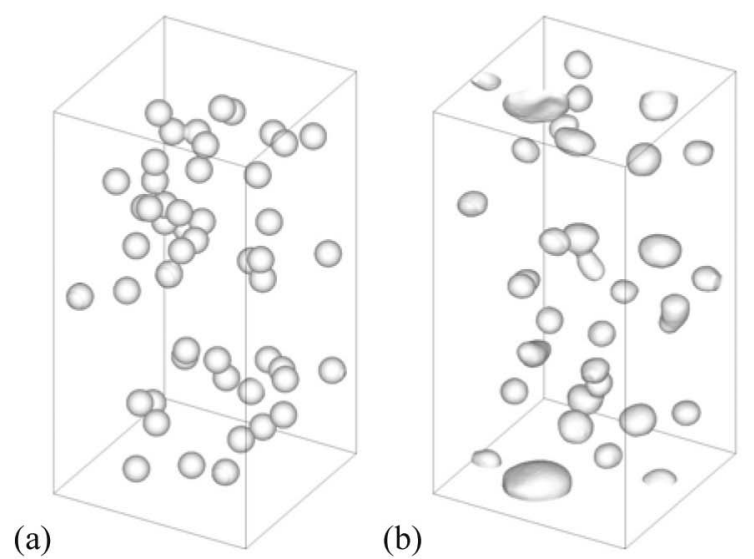

Fig. 9. Bubble Shapes at (a) Time $=0.00$, (c)

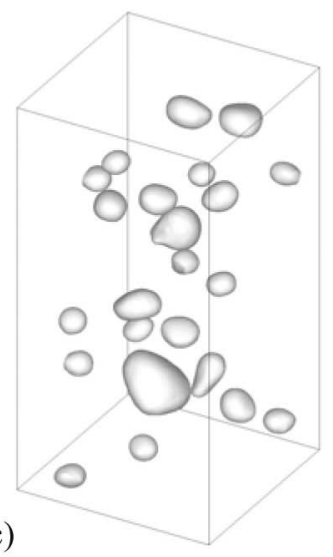

(d)

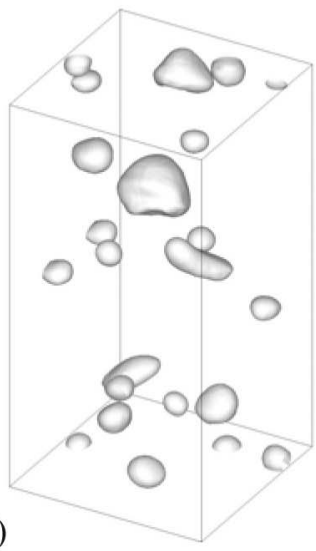

(b) 0.12 , (c) 0.24 , and (d) 0.36 Seconds

decreases. Although the result is not compared with the experimental data, this calculation demonstrates the robustness of the CIP-CSL2 method for a complicated area of the free-surface calculation.

\section{HEAT AND MASS TRANSFER MODELING WITH PHASE-FIELD APPROACH}

Our PF model belongs to the family of diffuse interface formulations; the main characteristic that sets this model apart is the treatment of the phase change. In this case, the location of any interface is determined by the variation of a smooth phase indicator field (or simply PF), which denotes the state of matter (liquid, solid, gas, degree of order, magnetization, crystallographic structure, etc). Based on the distribution of the indicator field, we can obtain the free energy of the system through the introduction of a phenomenological functional as:

$$
F=\int_{\Omega}\left\{f(\varphi)+\frac{1}{2} \lambda(\nabla \varphi)^{2}\right\} d \Omega,
$$

where $f$ is the homogeneous part of the free energy, $\varphi$ the $\mathrm{PF}$ and $\lambda$ a positive parameter that might be a function of the PF, temperature and other fields. This functional possesses the particularity that any change in the phase indicator field must decrease the free energy; therefore, it represents mathematically the intuitive idea that "any process in nature takes place to minimize the energy of a system", which goes along with the second law of thermodynamics.
The first term in the integrand corresponds to the homogeneous free energy density and the second one represents the energy contribution arising from the presence of an interface. To discretize this functional, we have to use several grid points over the interface between phases to resolve correctly the derivatives of the PF, which imposes a severe constraint on this kind of model. For instance, the real interface thickness of a liquid vapor interface at relatively high temperature should be of the order of one nanometer [45], meaning that we must use a grid spacing smaller than that inside the diffuse interface to evaluate the inhomogeneous term correctly. In phenomenological $\mathrm{PF}$ formulations, this constraint is loosened and changed for another one, numerically more friendly, in which the interface thickness is artificially increased. Nonetheless, this enlarged interface thickness introduces some spurious effects on the transport equations. These spurious effects can be determined by performing a thin interface analysis of the model, and then eliminated with the right choice of PF parameters. Hence, the new constraint only demands that the artificially enlarged interface thickness be smaller than the local curvature or any other characteristic length that controls the dynamic of the interface. These ideas have already been used successfully in dendritic growth [46] and solid state phase transitions [47], and now we are aiming to export these concepts to boiling. Once the free energy functional of a system has been established, the evolution of a non-conserved order parameter will follow a relaxation process, which can be expressed mathematically as:

$$
\frac{\partial \varphi}{\partial t}=M(\varphi) \frac{\delta F}{\delta \varphi}
$$




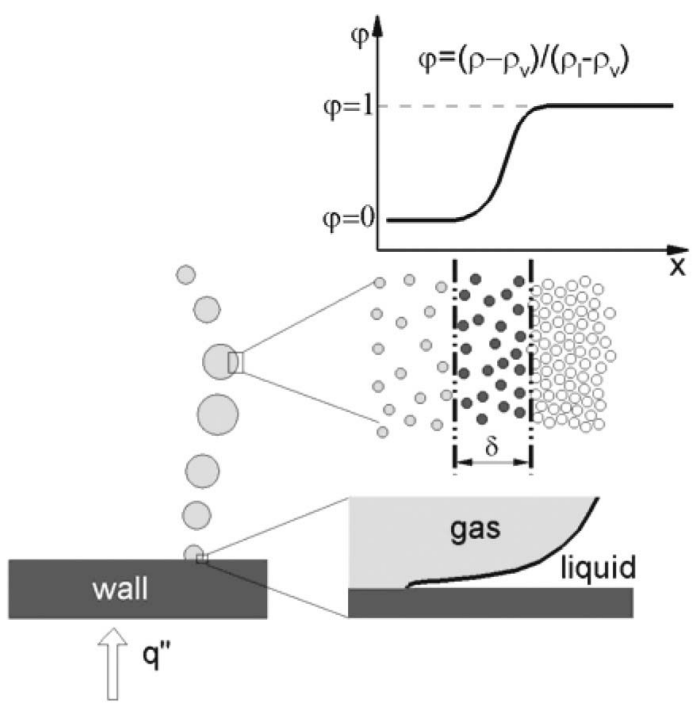

Fig 10. Schematic of the Phase-Field Model for Boiling

where $M(\varphi)$ is a positive parameter and $\delta F / \delta \varphi$ represents the transformation potential (usually miscalled the chemical potential). When expressions (15) and (16) are coupled to the energy, mass and momentum equations, we can develop a meso-scopic model for simulating diffusion controlled growth processes, such as the growth of a bubble immersed in a superheated liquid. A schematic of the boiling process and the meaning of the PF are presented in Figure 10.

\subsection{Results}

Numerical simulations of a two-dimensional bubble growing in a superheated liquid have been performed with PF and CIP-CLS2 to assess the performance of these two models. A bubble with an initial diameter of $7 \mathrm{~mm}$ was placed at the center of a square cavity and allowed to grow due to heat diffusion (see Figure 11). The properties of both phases (liquid and vapor) were set equal to evaluate the magnitude of the parasitic currents. For a case with a high density ratio (i.e. water and vapor), the velocity field originated from the liquid expansion, hide the parasitic currents making this assessment more difficult. The physical properties used in the simulations are presented in Table 1. In the case of CIP-CLS2 simulations, the temperature of the vapor was set to the saturation value and the evaporation rate was directly obtained from:

$$
\ddot{m}=k \nabla T \cdot \nabla \varphi / L
$$

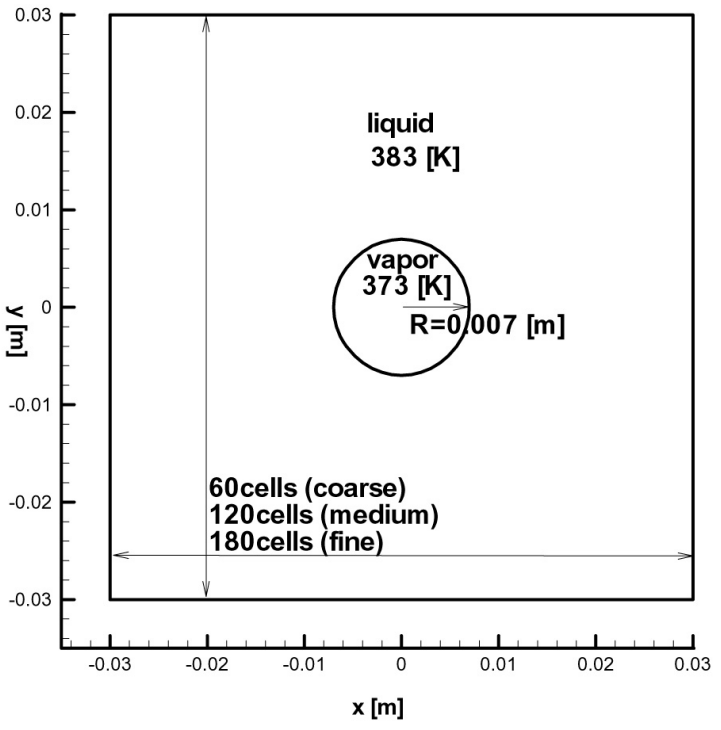

Fig 11. Two Dimensional Test Case of a Bubble Growing in A Superheated Liquid (Constant Properties and Equal in Both Phases)

Table 1. Physical Properties

\begin{tabular}{c|c|c|c|c|c}
\hline & $k$ & $\rho$ & $c_{p}$ & $\mu$ & $L$ \\
\hline \hline Vapor & 10 & 1 & 1000 & 1 & 20000 \\
\hline Liquid & 10 & 1 & 1000 & 1 & Thermal conductivity $[\mathrm{W} / \mathrm{K} \cdot \mathrm{m}]$ \\
\hline$k$ & \multicolumn{4}{c}{ Density $\left[\mathrm{kg} / \mathrm{m}^{3}\right]$} \\
\hline$\rho$ & \multicolumn{4}{c}{ Deat capacity $[\mathrm{J} / \mathrm{K} \cdot \mathrm{kg}]$} \\
\hline$c_{p}$ & \multicolumn{4}{c}{ Latent heat $[\mathrm{J} / \mathrm{kg}]$} \\
\hline$\mu$ & \multicolumn{5}{c}{$c$}
\end{tabular}

where $k$ is the thermal conductivity and $L$ the latent heat per unit mass. This expression does not correspond to a sharp interface formulation and consequently its accuracy is expected to be highly dependent on the grid spacing. This was clearly observed in the numerical results (Figure 12), in which the position of the interface as well as the temperature distribution was not independent from the grid size. In this case, PF simulations showed better performance in terms of grid independence. As for the intensity of the parasitic currents, PF simulations also showed better results than those of the mechanistic model. 

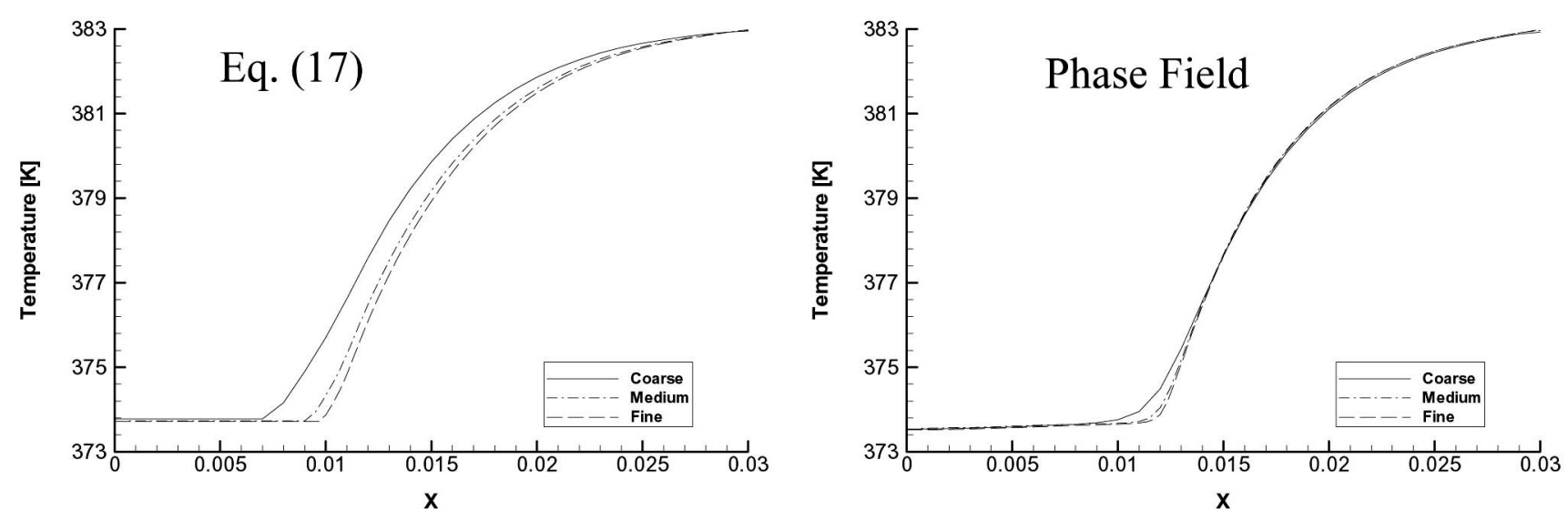

Fig. 12. Temperature Profiles Plotted From the Center of the Bubble

Figure 13 presents the maximum value of the velocity field as a function of the grid index, which is defined as:

$$
\text { Grid index }=\frac{\Delta x}{\Delta x_{\text {fine }}}
$$

It is important to remark that these results are only preliminary. The new version of the sharp interface model for calculating the evaporation rate has been shown to be

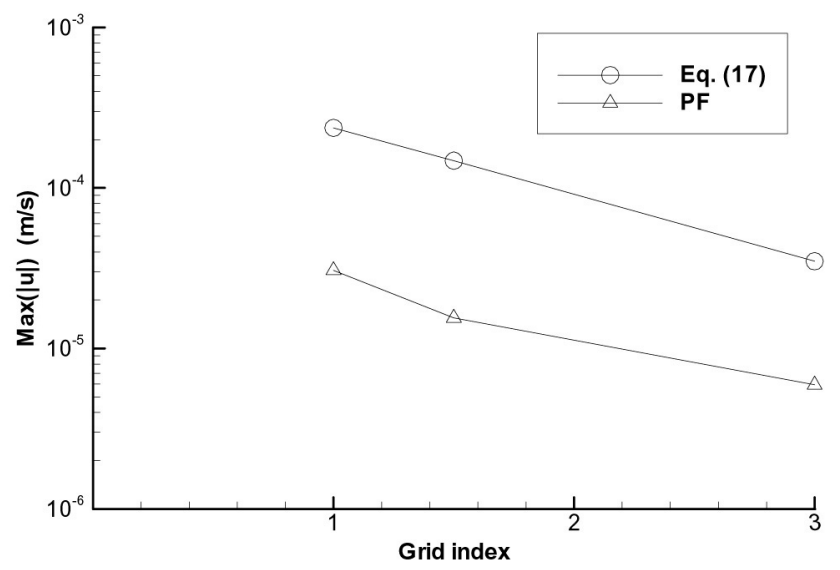

Fig 13. Parasitic Currents Intensity for Mechanistic and PF Simulations highly accurate in one dimensional simulations. A final comparison between the two models will be carried out once the implementation of the sharp interface model in three dimensions is finished.

\section{COUPLING OF DIFFERENT SCALES AND METHODOLOGIES}

In the framework of this project, in which four different scales can be considered, we need three couplings, which we can call links for convenience. Ordered from larger to smaller scales, the links are present between:

1. macro- and meso-scales,

2. meso- and micro-scales, and

3. micro- and nano-scales.

\subsection{Link Between Macro- and Meso-Scales}

The link between macro and meso-scales is the final goal of this project. It will comprise a set of physically based closure laws. The exact form of these laws will depend on the type of simulation being performed at the macro-scale. If macro-scale simulations are performed using the RANS based turbulence model with a two-fluid description of the flow field, meso-scales have to provide averaged heat release, void creation at fuel rod bundles and friction at the walls in the boiling regime. If, on the other hand, macro-scales will be analyzed with the LES approach and explicit bubble tracking, we would need unsteady information from the meso-scales, such as frequency of vapor bubble occurrence and detachment, instantaneous heat releases associated with that, and instantaneous wall friction. This link will be established in the next stage of the project. 


\subsection{Link Between Meso- and Micro-Scales}

This link is the current focus of our research, for which we are exploring two different options: using the same methodology employed for both micro- and mesoscales, or using different approaches for each scale. In the former case, we would establish a robust link between the scales. PF models offer the possibility of describing both scales and even allow simultaneous simulations, but such a strong link might not be optimal. Resolution requirements imposed by the $\mathrm{PF}$ approach may be stricter than those of DNS, particularly in the area around the contact line as well as in the thermal boundary layer around each bubble, thus making such an approach impractical. Besides, surface tracking techniques based on the Phase Field approach are not as accurate as the CIP-CSL2 algorithms.

It is therefore more probable that we will restrict the usage of the PF approach to the micro-scales, i.e. to the accurate modeling of the contact line and supply the meso-scale with a model for the contact angle and mass evaporation rate. Although the meso-scales themselves will be treated with the CIP-CSL2, some important features from PF, such as the interface sharpening mechanism and the evaluation of the surface tension, could still be used.

\subsection{Link Between Micro- and Nano-Scales}

To accurately represent the phenomena at micro-scales, we should improve our understanding of the physical phenomena taking place at the smallest length and time scales. For instance, the evaporation of a pure liquid at the contact line is far from being well understood. Although some important attempts have been made to develop a model to predict the evaporation rate and the heat flux in this region $[29,48]$, the number of simplifying assumptions used in the mathematical development blurs their quantitative capabilities. For example, the variation of interfacial and bulk properties in the proximity of the wall could play an important role in the evaporation phenomena taking place at the contact line, but this has never been accounted for. Since the experimental techniques to measure fundamental variables, such as temperature, pressure and velocity inside the micro-region, have not yet been developed (and most probably will never be), we believe that MD simulations could provide a unique framework to improve current mechanistic models and further develop the PF approach for simulating evaporation phenomena in the micro-region.

\section{CONCLUSIONS AND OUTLOOK}

The long term program MSMA aims to develop a comprehensive computational framework for modeling convective boiling and, eventually, CHF. A multi-scale approach has been chosen to take into account the existence of a variety of phenomena that are controlled by widely different length scales. This approach is first developed for conditions that are relevant for subcooled boiling and, therefore, for the DNB type of CHF. In the future, these methods will be applied to more general conditions. This paper reports on the first phase of the project, in which the foundations of the computational structure have been laid.

The general structure of the project includes the development of a DNS tool supplemented by a module for interface tracking for addressing the meso-scale phenomena at the scale of a single bubble, techniques to address micro- and nano-scale processes, and the coupling to a CFD tool, which will simulate the global boiling processes in the flow channels using information from the smaller scales. The first phase of the project has been focused on developing the most appropriate modeling and numerical techniques for simulating meso- and micro-scale phenomena, which control the growth of bubbles from the wall and the details of heat transfer and contact line dynamics, respectively.

A first important result has been achieved with the development of a highly efficient DNS solver, the PSIBOIL code, which provides the back-bone of the entire computational framework. The code has already been extensively validated, and some examples have been presented.

The selection of appropriate tools for the micro-scale and the coupling between meso- and micro-scales is the most challenging topic of the current research. In particular, the accurate simulation of the motion of the water-steam interface due to phase change and numerical issues such as mass conservation deserves special attention. It is important to point out that the aim of this research is to develop techniques that are suitable for simulating convective flow conditions, water as working fluid, rod bundle geometry, and the entire range of pressure that is of interest in nuclear reactor technology. Therefore, no short-cuts such as prescribed bubble shape or restricting the applicability of the codes to simpler fluids will be adopted. In consideration of this ambitious goal, the research proceeds in a step-by-step fashion, with various techniques explored. Two main lines of development are followed: coupling of an accurate interface tracking technique to a semi-empirical model for the micro-region, and a unified approach, based on the application of the phase-field method. Some initial results using both techniques have been presented, which show the merits and limitations of both concepts. Initially, the two approaches were supposed to be in competition and the best method was to be selected. However, in consideration of the results obtained, difficulties that have been recognized and, in general, the in-depth understanding gained from the initial application of the methods, the general strategy for the coupling between the meso- and the micro-scale is currently being re-evaluated. In particular, the two approaches may eventually be used together to 
provide different pieces of information on the evaporation process and the hydrodynamics of the contact line. Additionally, it has been recognized that for the simulation of the details of the evaporation process, information at the nano-scale on the basic properties of an ultra-thin liquid film may be required to close the equations describing the evaporation of the liquid micro-layer. Therefore, for an accurate treatment of the evaporation process at the triple interline, information obtained from MD may result to be necessary for closing the equations describing the heat transfer process. Preliminary research activities to address these aspects will be undertaken soon, and more efforts on this topic will be made in a future stage of the program. In consideration of the uncertainties regarding the importance of the nano-scale effects, the simulation approach for the meso and micro-scale will be optimized to produce the most accurate and numerically efficient representation of the life cycle of a bubble observed in convective boiling experiments. The successful prediction of the hydrodynamics of an individual bubble, and a reasonable estimate of the associated heat transfer processes, are thus the goals of the first stage of this research. An important aspect of this first validation exercise will also be the evaluation of the impact of a simplified treatment of the micro-scale phenomena on the accuracy of the calculation of the wall heat transfer rate.

In the next stage of the program, the importance of the effects of nano-scale processes on the global boiling heat transfer will be evaluated and additional submodels will be introduced to refine the calculation of heat transfer. MD simulations will be used to guide these developments. On the other end of the spectrum of length scales, the next stage of the program will also deal with the coupling of the smaller scales to the macro-scale. Finally, a complete computational structure will be established, which will first be set and validated for low heat flux conditions. Later, it will be extended for application to higher heat fluxes and thermal crisis conditions.

The goal of the MSMA project is the development of a complete computational framework for simulating boiling processes and CHF. However, it is clear that the validation of the methods cannot rely only on the comparison with global experimental information, such as wall heat fluxes or CHF values. To validate the models, but also to guide their development, experimental information will be needed for variables such as liquid micro-layer thickness, details on bubble shapes, etc., for conditions close to those prototypical in a nuclear reactor core. The MSMA project is therefore intending to provide the scientific and technical motivation for a new class of experiments with advanced instrumentation, which will provide the information required for a fully mechanistic description of the boiling process. Although a very limited preliminary experimental activity is currently associated with the project, it is expected that MSMA will be the seed for a long-term, combined theoretical and experimental program. This program will primarily deal with boiling processes, but in consideration of the expected advances in the modeling of thin films and the detailed description of moving interfaces with phase change, the computational tools can also be used for a detailed description of other processes of technological interest (e.g., film condensation)

\section{ACKNOWLEDGEMENTS}

The authors are grateful to other members of the Laboratory for Thermal-Hydraulics, Paul Scherrer Institute, who helped with the preparation of the MSMA project proposal. In alphabetical order, thanks are due to: D. Paladino, H-M. Prasser and B.L. Smith.

\section{REFERENCES}

[1] IAEA. Renaissance is in motion. 2010 [cited; Available from:http://www.world-nuclear-news.org/nn_laea renaissance_is_in_motion_0609102.html.

[2] Stosic, Z.V. The nuclear renaissance and AREVA's reactor designs for the 21st century: EPR and SWR-1000. in International Conference Nuclear Energy for New Europe. 2007. Portorož, Slovenia.

[3] Guelfi, A., et al., Advanced boiling two-phase flow modeling: A major challenge for nuclear industry", in AIRH-SHF-SFT Colloquium on two-phase boiling flow. 2008: Grenoble, France.

[4] Smith, B., Lawrence, Assessment of CFD codes used in nuclear reactor safety simulations. Nuclear Engineering and Technology, 2010. 42(4): p. 339 - 364.

[ 5 ] Frank, T., C. Lifante, and A.D. Burns, Recent advances in modeling and simulation of boiling processes in ANSYS $C F D$, in ANSYS Conf. and 27th CADFEM Users Meeting. 2009: Leipzig, Germany.

[6] Ustinenko, V., et al., Validation of CFD-BWR, a new twophase computational fluid dynamics model for boiling water reactor analysis. Nuclear Engineering and Design, 2008. 238(3): p. 660-670.

[7] Wehle, F., et al., Progress in AREVA NP's LWR thermal hydraulics methodology, in Workshop on Modeling and Measurements of Two-Phase Flows and Heat Transfer in Nuclear Fuel Assemblies. 2006: Stockholm, Sweeden.

[ 8 ] Le Corre, J.-M., S.-C. Yao, and C.H. Amon, A mechanistic model of critical heat flux under subcooled flow boiling conditions for application to one- and three-dimensional computer codes. Nuclear Engineering and Design, 2010. 240(2): p. 235-244.

[9] Podowski, M.Z. and R.M. Podowski, Mechanistic multidimensional modeling of forced convection boiling heat transfer. Science and Technology of Nuclear Installations, Article ID 387020, 2009: p. 10.

[10] Bestion, D., et al., Review of available data for validation of NURESIM two-phase CFD software applied to CHF investigations. Science and Technology of Nuclear Installations, Article ID 214512, 2009: p. 14.

[11] Guelfi, A., et al., NEPTUNE: A new software platform for advanced nuclear thermal hydraulics. Nuclear Science and Engineering, 2007. 156: p. 281 - 324. 
[12] Jamet, D., et al. Towards a multi-scale approach of twophase flow modeling in the context of DNB modeling. in OECD/NEA Workshop on Experiments and CFD code Application to Nuclear Reactor Safety (XCFD4NRS). 2008. Grenoble, France.

[13] Koncar, B. and I. Tiselj, Influence of near-wall modelling on boiling flow simulation. Nuclear Engineering and Design, 2010. 240(2): p. 275-283.

[14] Ahn, H.S., et al., Pool boiling CHF enhancement by micro/nanoscale modification of zircaloy-4 surface. Nuclear Engineering and Design. In Press, Corrected Proof.

[15] Theofanous, T.G. and T.N. Dinh, High heat flux boiling and burnout as microphysical phenomena: mounting evidence and opportunities. Multiphase Science and Technology, 2006. 18(1): p. 1 - 26.

[16] Hirt, C.W. and B.D. Nichols, Volume of fluid (VOF) method for the dynamics of free boundaries. Journal of Computational Physics, 1981. 39(1): p. 201-225.

[17] Sussman, M., P. Smereka, and S. Osher, A Level set approach for computing solutions to incompressible twophase flow. Journal of Computational Physics, 1994. 114(1): p. 146-159.

[18] Peng, D., et al., A PDE-basedfast local level set method. Journal of Computational Physics, 1999. 155(2): p. 410-438.

[19] Enright, D., et al., A hybrid particle level set method for improved interface capturing. Journal of Computational Physics, 2002. 183(1): p. 83-116.

[20] Youngs, D.L., Time-dependent multi-material flow with large fluid distortion. In Numerical Methods for Fluid Dynamics. Numerical Methods for Fluid Dynamics, ed. K.W. Morton and M.J. Baines. 1982, New York: Academic Press. 273-285.

[21] Sussman, M. and E.G. Puckett, A coupled level set and volume-of-fluid method for computing $3 D$ and axisymmetric incompressible two-phase flows. Journal of Computational Physics, 2000. 162(2): p. 301-337.

[22] Sussman, M., A second order coupled level set and volumeof-fluid method for computing growth and collapse of vapor bubbles. Journal of Computational Physics, 2003. 187(1): p. 110-136.

[23] Nakamura, T., et al., Exactly conservative semi-Lagrangian scheme for multi-dimensional hyperbolic equations with directional splitting technique. Journal of Computational Physics, 2001. 174(1): p. 171-207.

[24] Takewaki, H. and T. Yabe, The cubic-interpolated pseudo particle (CIP) method: application to nonlinear and multidimensional hyperbolic equations. Journal of Computational Physics, 1987. 70(2): p. 355-372.

[25] Yabe, T. and T. Aoki, A universal solver for hyperbolic equations by cubic-polynomial interpolation I. Onedimensional solver. Computer Physics Communications, 1991. 66(2-3): p. 219-232.

[26] Gibbs, J.W., On the equilibrium of heterogeneous substances. Connecticut Academy Transactions, 1877. 3: p. 343.

[27] Cahn, J.W. and J.E. Hilliard, Free energy of a nonuniform system. I. interfacial free energy. Journal of Chemical Physics, 1958. 28(2): p. 258 - 267.

[28] Ginzburg, V.L. and L.D. Landau, On the theory of superconductivity. Zhurnal Eksperimentalnoye $\mathrm{i}$ Teoreticheskoye Fiziki, 1950. 20: p. 1064.
[29] Lay, J.H. and V.K. Dhir, Shape of a vapor stem during nucleate boiling of saturated liquids. Journal of Heat Transfer 1995. 117(2): p. 394 - 401.

[30] Chorin, A.J., Numerical Solution of Navier-Stokes Equations. Mathematics of Computation, 1968. 22(104): p. $745-762$.

[31] Versteeg, H.K. and W. Malalasekera, An introduction to computational fluid dynamics The finite volume method. $2^{\text {nd }}$ ed. 2007: Prenticel Hall.

[32] Hutchinson, B.R. and G.D. Raithby, A multigrid method based on the additive correction strategy. Numerical Heat Transfer, Part B, 1986. 9: p. 27.

[33] Kuhn, S., Transport mechanisms in mixed convective flow over complex surfaces. 2008, Swiss Federal Institute of Technology (ETH): Zurich.

[34] Cherukat, P., et al., Direct Numerical Simulation of a Fully Developed Turbulent Flow over a Wavy Wall. Theoretical and Computational Fluid Dynamics, 1998. 11(2): p. 109-134.

[35] Henn, D.S. and R.I. Sykes, Large-eddy simulation of flow over wavy surfaces. 1999, Cambridge Journals Online. p. $75-112$.

[36] Xiao, F. and T. Yabe, Completely conservative and oscillationless semi-lagrangian schemes for advection Transportation. Journal of Computational Physics, 2001. 170(2): p. 498-522.

[37] Tanaka, R., T. Nakamura, and T. Yabe, Constructing exactly conservative scheme in a non-conservative form. Computer Physics Communications, 2000. 126(3): p. 232-243.

[38] Yabe, T., et al., An exactly conservative semi-lagrangian scheme (CIP-CSL) in one dimension. Monthly Weather Review, 2001. 129(2): p. 332-344.

[39] Yabe, T., F. Xiao, and T. Utsumi, The Constrained Interpolation Profile Method for Multiphase Analysis. Journal of Computational Physics, 2001. 169(2): p. 556-593.

[40] Toda, K., Y. Ogata, and T. Yabe, Multi-dimensional conservative semi-Lagrangian method of characteristics CIP for the shallow water equations. Journal of Computational Physics, 2009. 228(13): p. 4917-4944.

[41] Yabe, T., et al., The next generation CIP as a conservative semi-Lagrangian solver for solid, liquid and gas. Journal of Computational and Applied Mathematics, 2002. 149(1): p. $267-277$.

[42] Xiao, F., T. Yabe, and T. Ito, Constructing oscillation preventing scheme for advection equation by rational function. Computer Physics Communications, 1996. 93(1): p. 1-12.

[43] Brackbill, J.U., D.B. Kothe, and C. Zemach, A continuum method for modeling surface tension. Journal of Computational Physics, 1992. 100(2): p. 335-354.

[44] Brereton, G. and D. Korotney, Coaxial and oblique coalescence of two rising bubbles. In Dynamics of Bubbles and Vortics Near a Free Surface. Vol. 119. 1991, New York: ASME.

[45] Shi, B., S. Sinha, and V. Dhir, K, Molecular dynamics simulation of the density and surface tension of water by particle-particle particle-mesh method. Journal of Chemical Physics, 2006. 124(204715): p. 7.

[46] Karma, A. and W.-J. Rappel, Quantitative phase-field modeling of dendritic growth in two and three dimensions. Physical Review E, 1998. 57(4): p. 4323. 
NICENO et al., Multi-Scale Modeling and Analysis of Convective Boiling: Towards the Prediction of CHF in Rod Bundles

[47] Chen, L.-Q., Phase-field models for microstructure evolution. Annual Review of Materials Research, 2002. 32(1): p. 113-140.
[48] Stephan, P. and J. Hammer, A new model for nucleate boiling heat transfer. Wärme- und Stoffübertragung, 1994. 30: p. 119-125. 